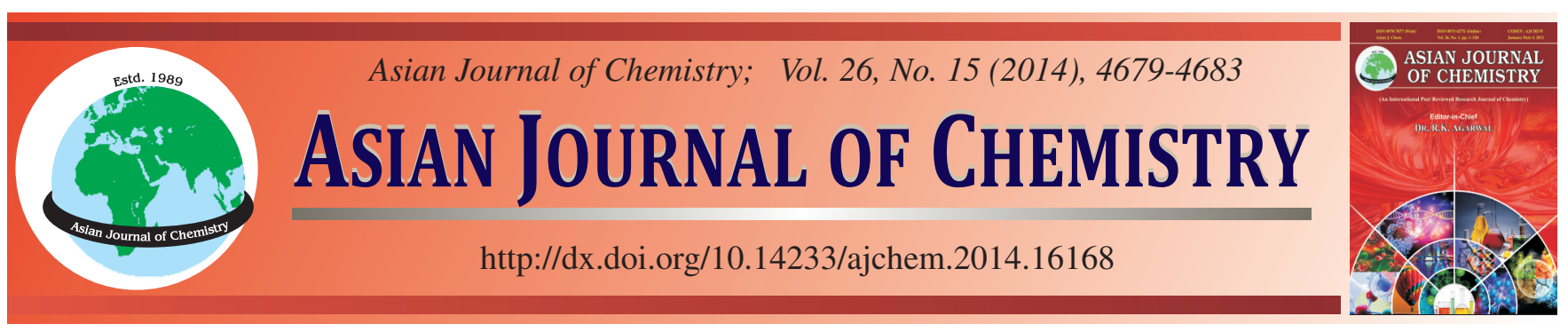

\title{
Nanoparticles-Polyaniline-Multiwalled Carbon Nanotubes for Determination of Organophosphate Pesticides
}

\author{
Min Wei*, JingJing Wang, JingXuAn GuO and Qiyu Lu*
}

College of Food Science and Technology, Henan University of Technology, Zhengzhou 450001, P.R. China

*Corresponding authors: E-mail: wei_min80@163.com

A sensitive, fast and stable amperometric acetylcholinesterase biosensor was developed based on gold nanoparticles-polyaniline-multiwalled carbon nanotubes (Au-PANI-MWCNTs) nanocomposite for quantitative determination of organophosphate pesticides. Because of the large surface areas and strong adsorptive ability of the Au-PANI-MWCNTs nanocomposite, the immobilized acetylcholinesterase developed high affinity for acetylthiocholine and exhibited excellent catalytic effect on the hydrolysis of acetylthiocholine. Based on the inhibition of organophosphorous pesticides on the activity of acetylcholinesterase, using parathion as a model compound, the conditions for detection of the pesticides were optimized. The inhibition of parathion was proportional to its concentration ranging from 0.2 to $2 \mathrm{nM}$ and 8 to $100 \mathrm{nM}$ with the correlation coefficients of 0.9982 and 0.9988 , respectively. The detection limit was $0.062 \mathrm{nM}$. The developed biosensor exhibited good reproducibility and acceptable stability.

Keywords: Acetylcholinesterase biosensor, Au-PANI-MWCNTs, Parathion.

\section{INTRODUCTION}

Organophosphorous pesticides (OPs) are extensively used in the agricultural industry due to their high insecticidal activity $^{1}$. However, organophosphorous pesticides are harmful for human health because they can reduce activity of acetylcholinesterase (AChE) and disturb the central nervous system in humans, leading to exhaustion, paralysis and ultimately death $^{2}$. In order to protect human health and environment, it is important to establish a rapid, sensitive method for determination of organophosphorous pesticides. The main traditional techniques for organophosphorous pesticides monitoring are chromatography, but these methods require professional operators, expensive instrumentation and complicated pretreatment steps and are not suitable for field inspection ${ }^{3}$. Electrochemical detection based on acetylcholinesterase biosensors are portable, less expensive and do not require tedious sample pretreatment, so they are regarded as highly promising candidates for on-site pesticide detection ${ }^{4}$.

Effective immobilization of enzyme to the electrode surface still remains a great challenge for the fabrication of biosensor ${ }^{5}$. In order to get high electron transfer rate and bioactivity of enzyme on the electrode surface, various kinds of nanomaterials have been employed in the area of biosensors, such as $\mathrm{CdS}^{6}$, carbon nanotube ${ }^{7-10}$, gold nanoparticles (AuNPs) ${ }^{11-13}$, silica sol-gel ${ }^{14,15}$, etc. Since gold nanoparticles have the capability to improve the conductivity of the electrode, facilitate the electron transfer and enhance the sensor selectivity and sensitivity, they have been successfully used to modify electrode for catalytic enlargement ${ }^{16-18}$. Multiwalled carbon nanotubes (MWCNTs) possess a lot of useful advantages such as large surface areas, strong adsorptive ability, excellent electrical conductivity and good biocompatibility, so they also have been used as effective catalyst supports in biosensors ${ }^{19-20}$. Due to its unique features with thermal stability, high chemical durability, good environmental stability and easy producibility, polyaniline (PANI), a kind of conducting polymer, has attracted a lot of attention ${ }^{21-26}$.

In this paper, we introduced Au-PANI-MWCNTs nanocomposite to immobilize acetylcholinesterase. Based on the inhibition of organophosphorous pesticides on the activity of acetylcholinesterase, using parathion as a model compound, the conditions for detection of the pesticides were optimized.

\section{EXPERIMENTAL}

Acetylcholinesterase (Type C3389, $500 \mathrm{U} / \mathrm{mg}$ from electric eel) and acetylthiocholine chloride were purchased from SigmaAldrich (St. Louis, USA) and used as received. Parathion-ethyl ( $\geq 99 \%$ ) was obtained from Augsburg (Germany). Bovine serum albumin (Solarbio, China), Glutaraldehyde (Kermel, China). All other chemicals were of analytical-reagent grade and used without further purification. Double distilled water was used throughout the experiments. 
All the electrochemical experiments are performed on a CHI 660D Electrochemical Workstation (Shanghai Chenhua Instrument Corporation, China). A three-electrode system comprising platinum wire as auxiliary electrode, saturated calomel electrode (SCE) as reference electrode and the modified or unmodified boron-doped diamond (BDD) as working electrode.

Preparation of Au-PANI-MWCNTs nanocomposite: PANI-MWCNTs nanocomposite was prepared according to the literature ${ }^{27}$. Au-PANI-MWCNTs nanocomposite was obtained by chemically reducing $\mathrm{AuCl}_{4}^{-}$on the positively charged PANIMWCNTs nanocomposite surface. Firstly, the prepared PANIMWCNTs nanocomposite was dispersed in Au colloid solution under stirring vigorously for several hours to allow complete adsorption of Au colloid onto the positively charged PANIMWCNTs nanocomposite surface. The Au colloid coated on PANI-MWCNTs nanocomposite surface serves as seeds for subsequent growth by electroless gold plating. After being rinsed repeatedly with deionized water, the Au-coated PANIMWCNTs nanocomposite dispersion were diluted to $20 \mathrm{~mL}$ with water, then $\mathrm{HAuCl}_{4}(1 \mathrm{wt} . \%, 0.3 \mathrm{~mL})$ and $\mathrm{NH}_{2} \mathrm{OH} \cdot \mathrm{HCl}$ $(0.04 \mathrm{M}, 1.2 \mathrm{~mL})$ were added under stirring for $15 \mathrm{~min}$ to increase and stabilize the amount of Au grown on the PANIMWCNTs nanocomposite.

Fabrication of acetylcholinesterase biosensor: Borondoped diamond was sequentially sonicated for $10 \mathrm{~min}$ in anhydrous ethanol, acetone and distilled water, then allowed to dry at room temperature. The modified electrode was prepared as follows: Firstly, 4 mg Au-PANI-MWCNTs nanocomposite was dispersed in $10 \mathrm{~mL}$ of distilled water by strongly stirring. Then $8 \mu \mathrm{L}$ acetylcholinesterase solution $(0.24 \mathrm{U}$, containing $1 \%$ BSA to maintain the stability of acetylcholinesterase and $0.5 \%$ glutaraldehyde) was mixed with $10 \mu \mathrm{L}$ of the Au-PANI-MWCNTs nanocomposite and sonicated thoroughly. The pretreated boron-doped diamond was modified by dropping $10 \mu \mathrm{L}$ of the mixture solution of acetylcholinesterase and Au-PANI-MWCNTs and allowed to be dried in the refrigerator $\left(4{ }^{\circ} \mathrm{C}\right)$. The obtained AChE/Au-PANIMWCNTs/BDD was stored at $4{ }^{\circ} \mathrm{C}$ when not in use.

Measurement procedure: For the measurement of parathion, the obtained AChE/Au-PANI-MWCNTs/BDD was first immersed in PBS ( $5 \mathrm{~mL}, \mathrm{pH}$ 7.5) solution containing $1 \mathrm{mM} \mathrm{ATCl}$ as substrate. After that, different concentration of standard parathion was added into the electrochemical cell to study the electrochemical response by differential pulse voltammetry (DPV). The inhibition of parathion is calculated as follows: inhibition $(\%)=\left[\left(\mathrm{I}_{0}-\mathrm{I}\right) / \mathrm{I}_{0}\right] \times 100 \%$. where $\mathrm{I}_{0}$ is the peak current of thiocholine, hydrolysis product of ATCl on the AChE/ $\mathrm{Au}-\mathrm{PANI}-\mathrm{MWCNTs} / \mathrm{BDD}$ and I is that with parathion inhibition.

Optimization of working electrode: The $\mathrm{pH}$ dependence of the biosensor response was studied in PBS at different $\mathrm{pH}$ of 6.0, 6.5, 7.0, 7.5 and 8.0. To study the effect of substrate concentration, different $\mathrm{ATCl}$ concentrations ranging from 0.05 to $1.2 \mathrm{mM}$ were tested by differential pulse voltammetry. The effect of different enzyme amount on the electrode response was tested in the range of $0.12 \mathrm{U}-0.28 \mathrm{U}$.

\section{RESULTS AND DISCUSSION}

Electrochemical impedance spectroscopy (EIS) study on different electrodes: Fig. 1 exhibits the Nyquist plot of
EIS for the different modified electrodes. The Nyquist plot of PANI-MWCNTs/BDD(Fig. 1b) and Au-MWNTs/BDD (Fig. 1c) electrode gave the Rct value of $462 \Omega, 572 \Omega$, respectively. The Au-PANI-MWCNTs/BDD electrode (Fig. 1a) possessed the lower Rct value of $335 \Omega$, implying that the Au-PANIMWCNTs had good synergistic effect to make electron transfer to the electrode surface easier. As shown in Fig. 1d, the Rct value increased to $818 \Omega$ for the AChE/Au-PANI-MWCNTs modified boron-doped diamond electrode, confirming the successful immobilization of acetylcholinesterase.

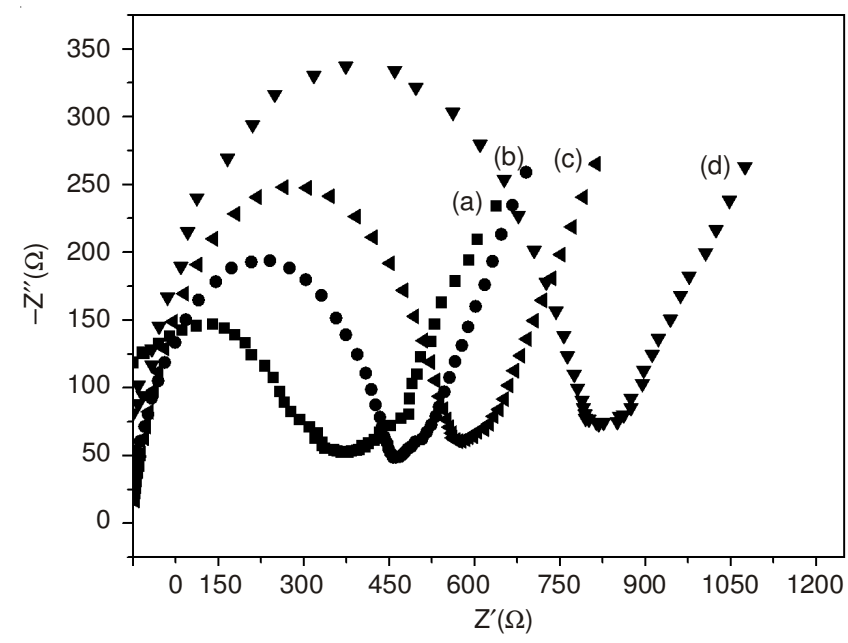

Fig. 1. Nyquist plot of EIS in $0.1 \mathrm{M} \mathrm{KCl}$ solution containing $1 \times 10^{-2} \mathrm{M}$ $\left[\mathrm{Fe}(\mathrm{CN})_{6}\right]^{3-14}$ at (a) Au-PANI-MWCNTs/BDD, (b) PANI-MWCNTs/ BDD, (c) Au-MWCNTs/BDD, (d) AChE/Au-PANI-MWCNTs/ BDD. Frequency range: $0.1 \mathrm{~Hz}$ to $10 \mathrm{kHz}$

Differential pulse voltammetry (DPV) response on different electrodes: The DPV response on different electrodes is shown in Fig. 2. As shown in Fig. 2a, no peak current was observed on AChE/Au-PANI-MWCNTs/BDD in pH 7.5 PBS. However, when $1 \mathrm{mM}$ ATCl was added into PBS, an obvious oxidation peak was showed on AChE/Au-PANI-MWCNTs/ BDD (Fig. 2b), whereas no detectable signal was observed on Au-PANI-MWCNTs/BDD without immobilization of acetylcholinesterase (Fig. 2c). Obviously, this peak came from the oxidation of thiocholine, hydrolysis product of acetylthiocholine, catalyzed by the immobilized acetylcholinesterase. Compared with the oxidation peak current of thiocholine at AChE/BDD (Fig. 2d), it can be seen that the response at AChE/ Au-PANI-MWCNTs/BDD was dramatically enhanced. This could be ascribed that the Au-PANI-MWCNTs nanocomposite could increase the surface area, promote acetylcholinesterase adsorption and enhanced the activity of acetylcholinesterase, so the immobilized acetylcholinesterase exhibited great affinity to its substrate and excellent catalytic effect on the hydrolysis of acetylthiocholine.

The produced current by thiocholine is related with the activity of immobilized acetylcholinesterase, which can be used as an indicator for quantitative measurement of the inhibition action of parathion on the activity of immobilized acetylcholinesterase. As shown in Fig. 3, when AChE/AuPANI-MWCNTs/BDD was immersed in $10 \mathrm{nM}$ parathion for $8 \mathrm{~min}$, the produced current by thiocholine decreased drastically, 


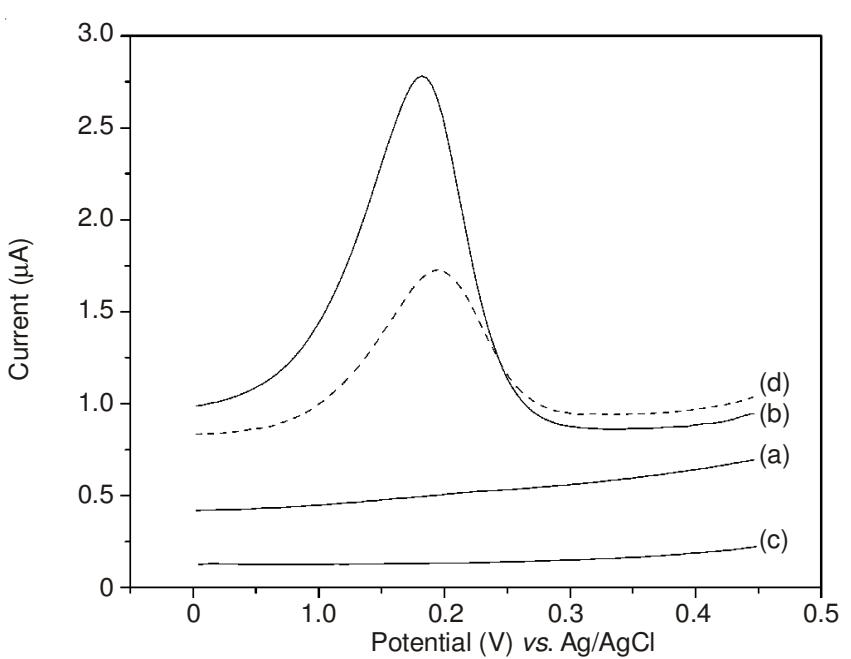

Fig. 2. (a) AChE/Au-PANI-MWCNTs/BDD in $\mathrm{pH} 7.5 \mathrm{PBS}$, (b) AChE/AuPANI-MWCNTs/BDD, (c) Au-PANI-MWCNTs/BDD and (d) AChE/BDD in $\mathrm{pH} 7.5$ PBS containing $1.0 \mathrm{mM} \mathrm{ATCl}$

as compared with that for $0 \mathrm{~min}$. This was because parathion, as one of the organophosphorous pesticides, exhibited fairly high acute toxicity and involved in the irreversible inhibition action on acetylcholinesterase, thus reduced the enzymatic activity. Due to the notable change in electrochemical signal of the AChE/Au-PANI-MWCNTs/BDD, the simple method for determination of parathion could be established.

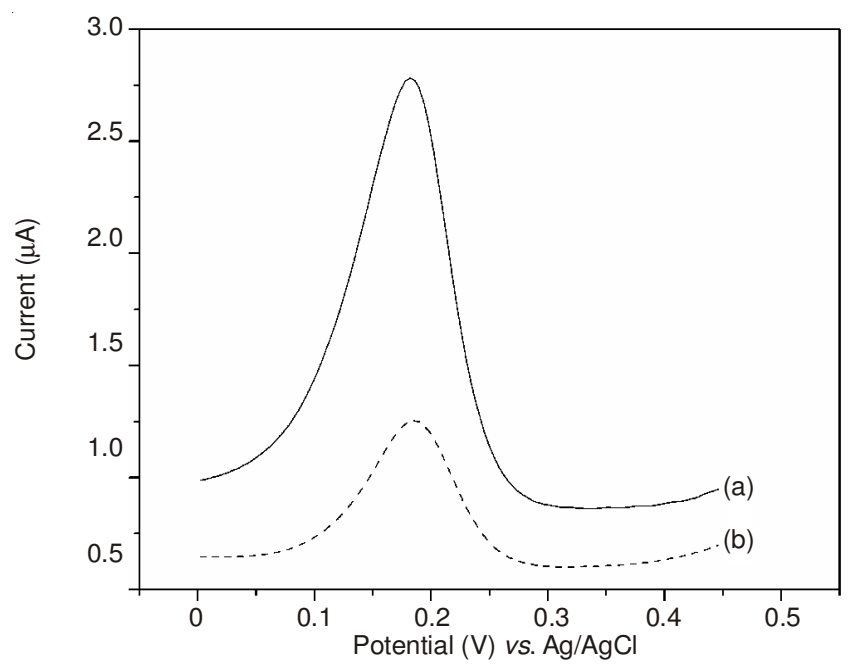

Fig. 3. DPV response of AChE/Au-PANI-MWCNTs/BDD in $\mathrm{pH} 7.5$ PBS containing $1 \mathrm{mM} \mathrm{ATCl}$ after inhibition in $10 \mathrm{nM}$ parathion solution for (a) 0 and (b) 8 min, respectively

Optimization of experimental parameters: The bioactivity of the immobilized acetylcholinesterase depended on the solution $\mathrm{pH}$. The $\mathrm{pH}$ dependence of the biosensor response in 0.1 M PBS from 6 to 8 was studied. Fig. 4A shows the relationship between the peak current and solution $\mathrm{pH}$. The response current increased from $\mathrm{pH} 6$ to 7 and obtained the maximum at $\mathrm{pH}$ 7.5. However, the enzyme lost activity irreversibly at higher $\mathrm{pH}$ values. Therefore, $\mathrm{PBS}$ of $\mathrm{pH} 7.5$ was selected for subsequent experiments.

Fig. 4B displays the effect of acetylcholinesterase loading on amperometric response. The DPV peak current increased with increasing amount of acetylcholinesterase and reached
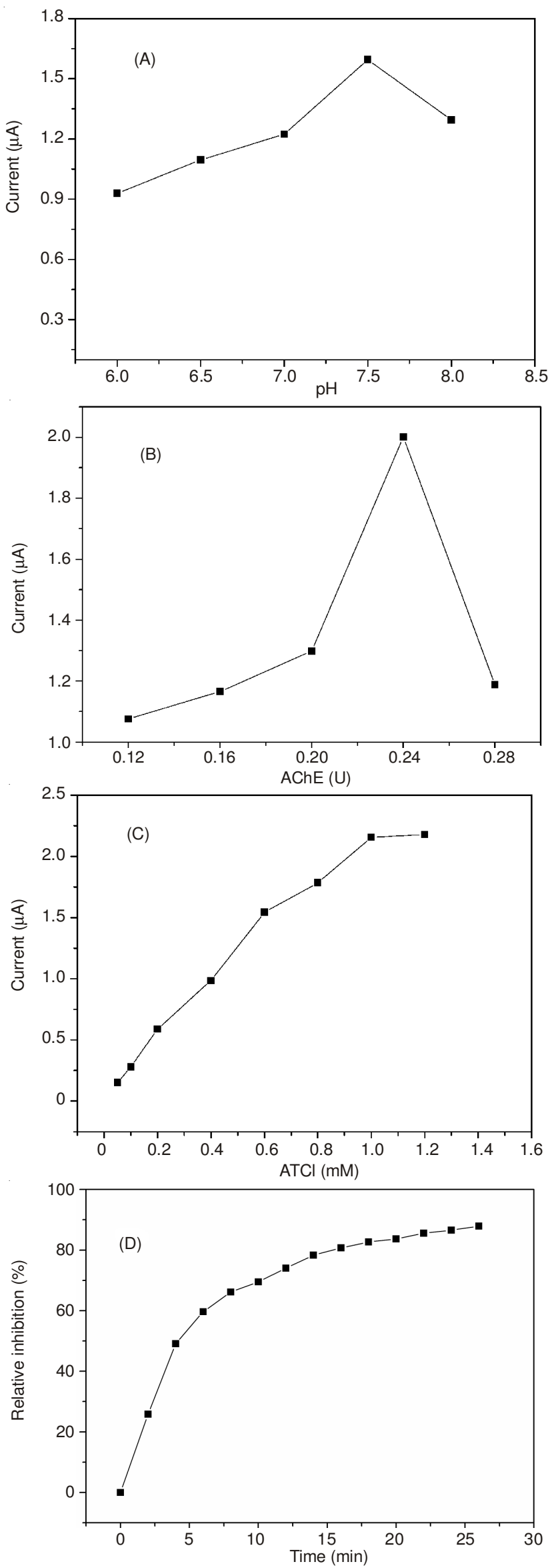

Fig. 4. Effects of $\mathrm{pH}(\mathrm{A}), \mathrm{AChE}$ loading (B), $\mathrm{ATCl}$ concentration (C) and inhibition time (D) on the response of AChE/Au-PANI-MWCNTs/ BDD biosensor in $0.1 \mathrm{M}$ PBS 
the maximum at $0.24 \mathrm{U}$, then decreased when the amount of acetylcholinesterase was increased further. This may be ascribed that the excess acetylcholinesterase could enhance the electrode resistance and slow the electron transfer between substrate and electrode ${ }^{28}$. So, $0.24 \mathrm{U}$ acetylcholinesterase (AChE ) was chosen as the optimal enzyme concentration, indicating saturation of enzyme loading.

The effect of acetylthiocholine concentration on the biosensor response from 0.05 to $1.2 \mathrm{mM}$ was also investigated. Fig. 4C shows the increasing trend in the current response with increasing acetylthiocholine concentration from 0.05 to $1 \mathrm{mM}$. There was no significant current improvement when the concentration exceeded $1 \mathrm{mM}$, which revealed that the electrode had reached its saturation level. From these results, $1 \mathrm{mM}$ acetylthiocholine was selected as optimum concentration for further experiments.

Fig. 4D displays the effect of inhibition time on the response of AChE/Au-PANI-MWCNTs/BDD. In the beginning, with the increase of inhibition time, the oxidation peak current on the biosensor decreased obviously and the inhibition rate increased accordingly. When the inhibition time is longer than $8 \mathrm{~min}$, the inhibition curve tends to a stable value, indicating the binding interaction between pesticides and active target groups in the enzyme reaches saturation. The inhibition rate was related with their interaction between pesticide and enzyme, which resulted in the change of the interactions with its substrate. However the maximum values of inhibitions was not $100 \%$, which was likely to attribute to the binding equilibrium between pesticide and binding sites in enzyme ${ }^{29}$.

Calibration curve: With increasing the concentration of parathion, the produced current by thiocholine on the AChE/ Au-PANI-MWCNTs/BDD decreased. In order to obtain a lower detection limit, an incubation time of 8 min was selected for inhibition measurements. Fig. 5 shows the inhibition curve, which has been obtained by plotting inhibition percentage (I \%) vs. parathion concentrations. Under the optimized experimental conditions, the parathion inhibition to AChE/Au-PANIMWCNTs/BDD is proportional to its concentration in two ranges, from 0.2 to $2 \mathrm{nM}$ and 8 to $100 \mathrm{nM}$. The linearization equations were inhibition $(\%)=19.188 x+20.735(\%)$ and inhibition $(\%)=0.1334 \mathrm{x}+65.297(\%)$, with the correlation coefficients of 0.9982 and 0.9988 , respectively. The detection limit was calculated to be about $0.062 \mathrm{nM}$, which is significantly lower than that of $1 \mathrm{nM}$ at MWCNT/Nafion film-modified glassy-carbon electrode ${ }^{30}$, indicating that the proposed Au-PANI-MWCNTs is suitable for the determination of organophosphorous pesticides.

Analysis of organophosphorous pesticides in apple skin samples: The content of parathion in real samples of apple skin was detected by AChE/Au-PANI-MWCNTs/BDD biosensor. A standard addition method was adopted to assess the reliability. As shown in Table-1, the recoveries for these apple skin samples obtained from the AChE/Au-PANI-MWCNTs/ BDD were found to be between 90.09 and $99.44 \%$, which indicated that this method could be used for assay of real samples.

Precision and stability: The intra-assay precision of the biosensor was calculated by assaying one enzyme electrode for eight replicate determinations in $1 \mathrm{mM}$ acetylthiocholine
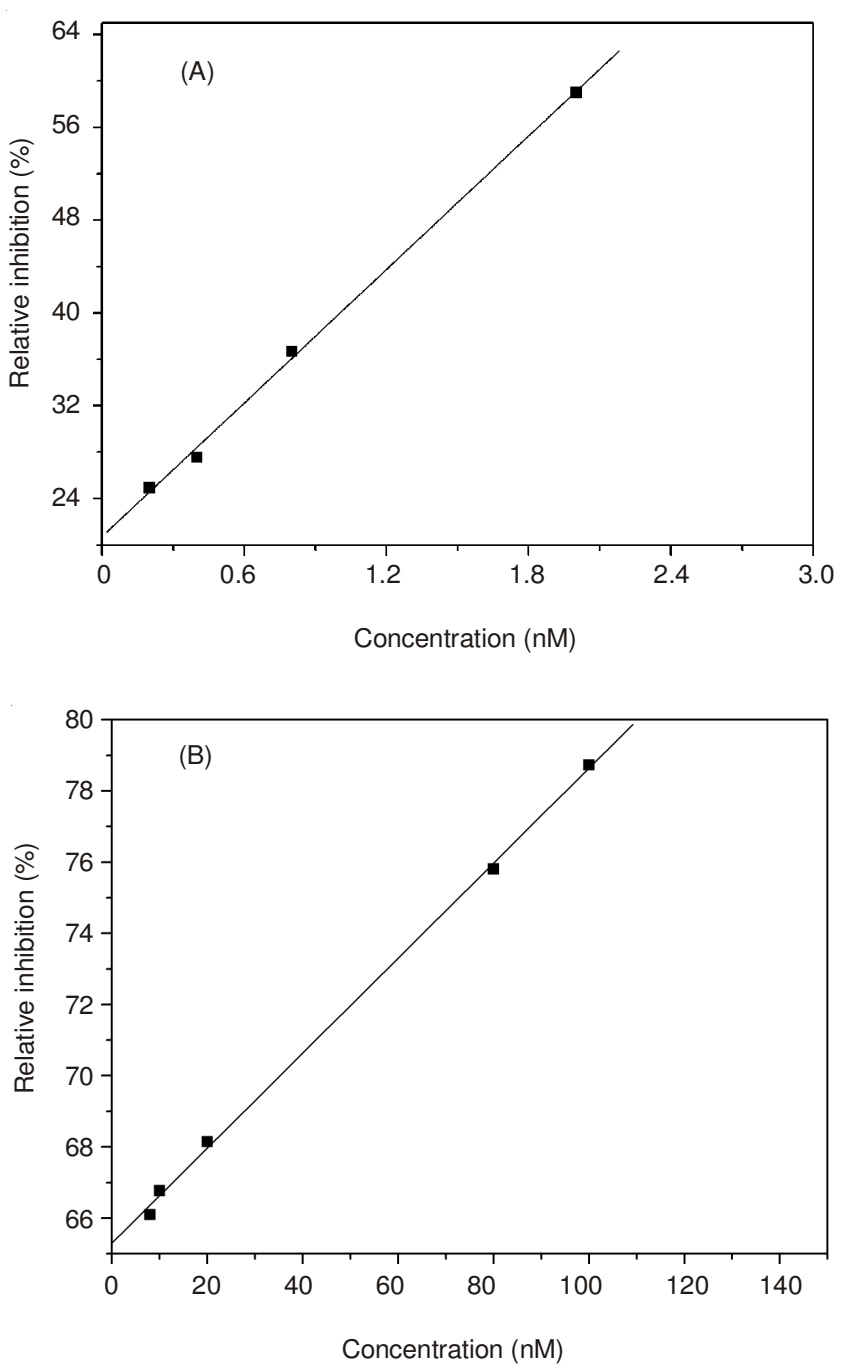

Fig. 5. Linear relationships between peak currents and parathion concentrations

\begin{tabular}{ccc}
\multicolumn{3}{c}{ TABLE-1 } \\
RECOVERY RATIOS OF PARATHION IN \\
APPLE SKIN SAMPLES \\
\hline $\begin{array}{c}\text { Added } \\
\text { concentration(nM) }\end{array}$ & $\begin{array}{c}\text { Concentration found } \\
(\mathrm{nM})\end{array}$ & Recovery $(\%)$ \\
\hline 0.54 & 0.537 & \\
1.82 & 1.785 & 99.44 \\
21.2 & 19.10 & 98.08 \\
&
\end{tabular}

after being treated with $10 \mathrm{nM}$ parathion for $8 \mathrm{~min}$ and the RSD was $4.93 \%$. When the AChE/Au-PANI-MWCNTs/BDD biosensor was not in use, it was stored in a refrigerator at $4{ }^{\circ} \mathrm{C}$. After a 30-day storage period, the sensor retained $94.63 \%$ of its initial current response, indicating the acceptable stability of biosensor.

\section{Conclusion}

A biosensor based on AChE/Au-PANI-MWCNTs/BDD was developed for determination of organophosphorous pesticides. The Au-PANI-MWCNTs nanocomposite could increase the surface area, promote acetylcholinesterase adsorption and enhanced the activity of acetylcholinesterase, so the biosensor exhibited higher sensitivity, lower detection limit, good repeatability and favorable stability toward organophosphorous pesticides detection. 


\section{ACKNOWLEDGEMENTS}

This research was supported by National Natural Science Foundation of China (Grant No. 21105022), Doctor Foundation of Henan University of Technology (2010BS019), Plan for Scientific Innovation Talent of Henan University of Technology (2012CXRC01), program for Science and Technology Development of Zhengzhou (20130876), Foundation for University Youth Key Teachers from Henan Province (2013GGJS-073) and Plan for Scientific Innovation Talent of Henan Province (124200510012).

\section{REFERENCES}

1. Y.-Y. Wei, Y. Li, Y.-H. Qu, F. Xiao, G.-Y. Shi and L.-T. Jin, Anal. Chim. Acta, 643, 13 (2009)

2. J.-Z. Zhang, A.-M. Luo, P. Liu, S.-P. Wei, G.-M. Wang and S.-Q. Wei, Anal. Sci., 25, 511 (2009).

3. V.B. Kandimalla and H. Ju, Chem. Eur. J., 12, 1074 (2006).

4. N. Chauhan, J. Narang and C.S. Pundir, Int. J. Biol. Macromol., 49, 923 (2011).

5. J.-M. Gong, L.-Y. Wang and L.-Z. Zhang, Biosens. Bioelectron., 24, 2285 (2009).

6. K. Wang, Q. Liu, L. Dai, J.-J. Yan, C. Ju, B.-J. Qiu and X.-Y. Wu, Anal. Chim. Acta, 695, 84 (2011).

7. H.-D. Chen, X.-L. Zuo, S. Su, Z.-Z. Tang, A.-B. Wu, S.-P. Song, D.-B. Zhang and C.-H. Fan, Analyst, 133, 1182 (2008).

8. Y.-H. Lin, F. Lu and J. Wang, Electroanalysis, 16, 145 (2004).

9. K.A. Joshi, J. Tang, R. Haddon, J. Wang, W. Chen and A. Mulchandani, Electroanalysis, 17, 54 (2005)

10. S. Viswanathan, H. Radecka and J. Radecki, Biosens. Bioelectron., 24, 2772 (2009).
11. D. Du, S.-Z. Chen, J. Cai and A.-D. Zhang, Biosens. Bioelectron., 23, 130 (2007).

12. H. Shi, L. Yuan, Y. Wu and S.Q. Liu, Biosens. Bioelectron., 26, 3788 (2011).

13. X. Cao, Y. Ye and S.Q. Liu, Anal. Biochem., 417, 1 (2011).

14. P. Raghu, T. Madhusudana Reddy, B.E. Kumara Swamy, B.N. Chandrashekar, K. Reddaiah and M. Sreedhar, J. Electroanal. Chem., 665, 76 (2012).

15. W. Wei, X. Zong, X. Wang, L. Yin, Y. Pu and S.Q. Liu, Food Chem., 135, 888 (2012).

16. J. Narang, N. Chauhan and C.S. Pundir, Analyst, 136, 4460 (2011).

17. Q.-Z. Yu, M.-M. Shi, Y.-A. Cheng, M. Wang and H.-Z. Chen, Nanotechnology, 19, 265702 (2008).

18. L. Bahshi, M. Frasconi, R. Tel-Vered, O. Yehezkeli and I. Willner, Anal. Chem., 80, 8253 (2008).

19. R. Rawal, S. Chawla, T. Dahiya and C.S. Pundir, Anal. Bioanal. Chem., 401, 2599 (2011)

20. N. Chauhan, J. Narang and C.S. Pundir, Analyst, 136, 1938 (2011).

21. D. Yang, Y. Ma, T.-Y. Zhai, Y. Zeng, H.-B. Fu and J.-N. Yao, Nanotechnology, 18, 1 (2007).

22. T.G. Kim, D. Ragupathy, A.I. Gopalan and K.P. Lee, Nanotechnology, 21, 134021 (2010).

23. L.-M. Guo and Z.-Q. Peng, Langmuir, 24, 8971 (2008).

24. N. Prabhakar, K. Arora, H. Singh and B.D. Malhotra, J. Phys. Chem. B, 112, 4808 (2008).

25. Q.-F. Chang, K. Zhao, X. Chen, M. Li and J. Liu, J. Mater. Sci., 43, 5861 (2008).

26. P. Santhosh, K.M. Manesh, S.-H. Lee, S. Uthayakumar, A.I. Gopalan and K.-P. Lee, Analyst, 136, 1557 (2011).

27. H.-Y. Lee, W. Vogel and P.P.-J. Chu, Langmuir, 27, 14654 (2011).

28. H.-S. Yin, S.-Y. Ai, J. Xu, W.J. Shi and L.-S. Zhu, J. Electroanal. Chem., 637, 21 (2009).

29. N. Chauhan and C.S. Pundir, Anal. Chim. Acta, 70, 66 (2011).

30. C.-Y. Li, C.-F. Wang, Y. Ma, W. Bao and S.-S. Hu, Anal. Bioanal. Chem., 381, 1049 (2005) 